\title{
Partial Quantum Tensors of Input and Output Connections
}

\author{
Andrew Dente \\ Independent Scholar, USA \\ Email: andyd180@yahoo.com
}

How to cite this paper: Dente, A. (2018) Partial Quantum Tensors of Input and Output Connections. Advances in Pure Mathematics, 8, 764-769.

https://doi.org/10.4236/apm.2018.88046

Received: July 4, 2018

Accepted: August 27, 2018

Published: August 30, 2018

Copyright (C) 2018 by author and Scientific Research Publishing Inc. This work is licensed under the Creative Commons Attribution International License (CC BY 4.0).

http://creativecommons.org/licenses/by/4.0/

\begin{abstract}
I show how many connections of $\Gamma$ are presently existing from $\mathbb{R}$ to $\beta$ as they are being inputted simultaneously through tensor products. I plan to address the Quantum state of this tensor connection step by step throughout the application presented. Also, I will show you how to prove that the connection is true for this tensor connection through its output method using a small bit of tensor calculus and mostly number theory.
\end{abstract}

\section{Keywords}

Tensors Products, Tensor Connections, Number Theory, Tensor Calculus, Input, Output, Quantum States

\section{Introduction}

Partial Quantum Tensors in summary, are network connections within the Quantum networks. These connections can be well understood using tensor calculus and even number theory. Tensor Calculus for one example, can be used to analyze the flow of electrons and to verify the movement within the electrons directions. Well, the reason why this application is called, "Partial Quantum Tensors" is because we only need to use partial methods within tensor calculus to analyze and verify the flow of Quantum input and output connections. The reason being is that Tensor Calculus can only verify the flow of particles or electrons that are perceptible through Euclidean space as this was first thought of by Neugebauer (1969) [1]. But in Quantum Networking, particles or electrons could be in two places at once. So how does that work? Well with Quantum connections we can't just use only tensor calculus to prove my application; we will have to use a reliable mathematical method that works well with Quantum mechanics, which will be number theory in this case. Number Theory combined 
with Tensor Calculus should give off effective results with this application because the many methods of Number Theory are extremely useful in relation to Quantum Mechanics such as the Riemann Zeta functions expressed within the Quantum Circuits. I will first start off this application by introducing several definitions to make this application come alive in the Quantum Networks.

\section{The Formulation of Quantum Networks}

1.0 Definition: The tensor connection $\Gamma^{2(\mathbb{R})} \beta$ holds a double input connection with $\Gamma^{2}$ clearly in conjunction with of course $\mathbb{R}$ and $\beta$.

1.0 Theorem: Now, we can only locate the connections through tensor processing of all individual connections $U$. Only then will we be able to send input signals $\Sigma$, to $\mathbb{R}$ and $\beta$.

1.1 Definition: Let $\otimes$ equals stable input connection and $\oplus$ equals unstable but working input connection. These are the main mechanisms for the tensor input connections.

1.2 Definition: Out of an arbitrary instance, $\gamma$ will indicate the best signal $\Sigma$ possible.

1.0 Proposition: Assume that $\varepsilon$ and $\xi$ are the Quantum Network areas that allow for a constant simultaneous connection for the tensor input methods.

1.3 Definition: We have to now assume that once our connection is in working order, we have to verify U's simultaneous tensor connections of $\mathbb{R}$ and $\beta$ for $\Sigma$ 's latency.

1.4 Definition: Let $\dagger$ and $\ddagger$ equal $\nabla$ and $\Delta$ if input connection results are precise.

1.5 Definition: Let $n$ substitute for $\Gamma$ if first connection is effective.

\section{Verifying the Flow of the Quantum Connections}

$$
\Gamma^{2(\mathbb{R})} \beta=\left\{\bigcup_{\Gamma=1}^{\infty} \frac{\Sigma^{(\mathbb{R} \otimes \beta)} \cdot \Sigma^{2}}{\gamma(\Sigma-\Sigma)}\right\}+\bigcup_{n=1}^{\infty} \underbrace{n^{\varepsilon}+2(\nabla)+\Sigma}_{n}=\frac{2 n^{\xi}}{\mathbb{R}+(\beta)_{\gamma} \dagger}
$$

As we can see from above in Expression (1), we used the tensor methods to find the stable connection between $\mathbb{R}$ and $\beta$. To make things more coherent, we used $\underbrace{}_{\sim}$ to carry out our mixed functional input connection and used $\nabla$ to balance both $\mathbb{R}$ and $\beta$ into the stable first wave of connections as presented above.

\section{Pinpointing the Network Groups}

We will be using tensor group theory to verify the result of the first wave of connections $\frac{2 n^{\xi}}{\mathbb{R}+(\beta)_{\gamma} \dagger}$ over $\mathbb{R}$ for $\Gamma^{2(\mathbb{R})} \beta$. Lo-keng Hua, Luogeng Hua, Yuan Wang, Springer-Verlag, (1981), made great contributions to understanding this mathematical method by using Number Theory to pinpoint the operator groups of numerical analysis's which we use today in Quantum Mechanics and 
such as this application [2]. M. Hassani (2004) helped us draw a path in understanding the connection made on network graphs by unique ways of using Number theory and Integer sequences combined [3]. Bombieri, Enrico (1990), helped form $\min / \max$ of bounded finite and infinite sets for Mordell revised conjecture which created new mathematics for understanding the limits and bounds of Quantum group sets and operators [4].

$$
\begin{gathered}
\Gamma^{2(\mathbb{R})} \beta=\sum_{n} \frac{n^{2}}{\beta}+\sum_{n}\left\{\sum_{n} \Gamma \frac{2 \nabla+\Sigma}{\frac{\xi^{2} \cdot(\gamma \otimes \mathbb{R})^{2}}{n-1}}+\sum_{\Gamma} \frac{\mathbb{R}-\xi}{\gamma^{2}+\Sigma}\right\}=\frac{\Sigma \cdot \Sigma^{(\beta \oplus \mathbb{R})}}{\gamma+\Sigma} \\
\frac{\Sigma \cdot \Sigma^{(\beta \oplus \mathbb{R})}}{\gamma+\Sigma}+\Gamma^{2(\mathbb{R})} \beta
\end{gathered}
$$

\section{Acquiring Network Efficiency}

1.1 Theorem: As seen in Expression (2) and (3), first wave of connections for the tensor product inputs yielded effective but not the best of tensor connections $\simeq$ due to there not being $\dagger$ but instead $\nabla$. First wave connection results: $\frac{\Sigma \cdot \Sigma^{(\beta \oplus \mathbb{R})}}{\gamma+\Sigma}=\mathbb{R}$ will have to overlap with the second wave connection results $\beta$ to gain a more precise input connection to verify that $\mathbb{R}$ of $\Gamma^{2(\mathbb{R})}$ will indeed benefit from the convergence of the second wave input connections $\beta$. Halmos, Paul (1974) first sought out an overview of this with his findings in vector calculus based off of finite dimensions [5]. We can see this working out precisely in Expression (4).

$$
\frac{\Sigma \cdot \Sigma^{(\beta \oplus \mathbb{R})}}{\gamma+\Sigma}+\Gamma^{2(\mathbb{R})} \beta \simeq \bigcup_{n=1}^{\infty} \underbrace{n^{\varepsilon}+2(\nabla)+\Sigma}_{n}
$$

\section{Input Connections Start to Conjoin Simultaneously}

1.2 Theorem: As the transition is taking place in Expression (4) and (5), $U$ will be redundant because all futile input connections have just been eradicated from the input source of the Quantum Network areas and will only be left with solving for the exact product of $\beta$ which in return will help with solving the exact connection of $\mathbb{R}$ or $\Gamma^{2(\mathbb{R})}$. Faltings, Gerd (1994), found that in algebraic geometry, algebra and geometry gave input to simultaneous expressions to explain elliptic systems that conjoined together [6]. Aguiar, M., Mahajan, S. (2010) used Monoidal Functors to understand The Schubert Statistic which in return helped us understand the inverse function of such connections that were made in his mathematical experiment and this also helped us form our input connection for the Quantum Networks [7].

$$
\Gamma^{2(\mathbb{R})} \beta=(\nabla)^{2} \prod_{n=1}^{\infty}\left\{\beta \cdot \gamma \frac{n-\Sigma}{\mathbb{R}+\xi}\right\}+2 \Gamma(\nabla)\left(\gamma_{n^{2}}-\mathbb{R}^{2}\right)^{\ddagger}+\beta
$$

Due to there being $\ddagger$ for both $\mathbb{R}$ and $\beta$ in Expression (5), means we 
have found a precise input connection for both first wave connections and second wave connections. Also, $\gamma$ over $\Sigma$ in our tensor input product verifies that both of these connections are indeed truly accurate.

$$
\begin{gathered}
\Gamma^{2(\mathbb{R})} \beta=\prod_{n=1}^{\infty}\left\{\frac{\Sigma(n)}{\mathbb{R}^{2}}\right\}+\Gamma(\beta) \\
\Gamma+[\beta]\left(\frac{\Sigma(n)}{\mathbb{R}^{2}}\right) \equiv \Gamma^{2(\mathbb{R})} \beta
\end{gathered}
$$

\section{Both Connections Verified}

1.0 Lemma: Expression (6) is unraveling to us how $\mathbb{R}$ benefited from $\beta$ and while Expression (7) is showing us how the effectiveness of the connections $n$ has remained constant throughout both first and second wave connections. Also, it's showing us that the first and second wave connection of $\mathbb{R}$ and $\beta$ do indeed have a precise verifiable simultaneous connection with the double input tensor connection $\Gamma^{2(\mathbb{R})} \beta$ that we have evaluated within the Quantum networks.

\section{Proving That the Verified Networks Are True}

To prove that the input connection is correct even further, we will trace it from the source of its output connection through: $\Gamma+[\beta]\left(\frac{\Sigma(n)}{\mathbb{R}^{2}}\right)$ with respect to $n$ and $\Gamma$ over $\Sigma$ for the output tensor method of integration for $\Gamma^{2(\mathbb{R})} \beta$. These results based on Quantum Mechanics can be scrutinized thoroughly in a similar manner using, (2007) Grillet Pierre's methods of abstract algebra where he helps us understand his advanced workings of group theory which then shaped the way we apply tensor calculus and number theory [8].

$$
\begin{gathered}
n=\int_{\Sigma} \Gamma(\beta) 2 \mathbb{R}\left\{n^{2}\right\} \Delta \frac{n}{\mathbb{R}^{2}}+\int_{\Sigma}\left\{\frac{\mathbb{R} \Delta}{\gamma^{2}+\beta}+\sum_{n=1}^{n} \frac{2 \gamma}{\xi^{2}+\varepsilon^{(\beta \cdot \mathbb{R})}}\right\} \\
=\left\{\int_{\Sigma} \sum_{n=1}^{n} \Delta\left(2 \xi_{\varepsilon}-n^{2+(\beta) \mathbb{R}}\right)\left[\frac{2-\gamma^{2}}{\mathbb{R} \Delta}\right]\right\} \mathrm{d} \Gamma
\end{gathered}
$$

1.1 Lemma: So far, the imbalance and balance between $\frac{n}{\mathbb{R}^{2}}$ of Expression (8) has yielded a semi stable number of connections for $n$ with respect to $\Gamma$ over $\Sigma$. Though $\beta$ second wave of output connections seem to be providing the most fluent output signals $\gamma$ with respect to the integration of $\Sigma$ while $\mathbb{R}$ is showing signs of balance $\Delta$ for its first wave of output connections but it does not have the best response signal for its output connection $\gamma$ as precisely shown in Expression (9). However, it's still showing signs as an effective output source in relation to its input connection described in Expression (7) due to $n$ being constant overall with $\mathbb{R}$ and which can be seen here unraveling in Expression (10). 


$$
n=\frac{n^{2}}{\mathbb{R}} \int_{\Sigma} \Delta \frac{\varepsilon_{2}+\gamma_{\xi}+\beta}{(n-2 \Delta)} \mathrm{d} \Gamma
$$

\section{Verifying the Output Source of the Input Connections}

1.3 Theorem: We can clearly see that $n$ is conjoining with $\beta$ to increase the output sources speed and fluency of $\mathbb{R}$ to indeed prove that: $\Gamma+[\beta]\left(\frac{\Sigma(n)}{\mathbb{R}^{2}}\right)$ input source measures the effectiveness of it's stable output source: $\frac{n^{2}}{\mathbb{R}}$ and to prove its original network connection $\Gamma^{2(\mathbb{R})} \beta$. This can be seen in Expression (10) and Expression (11).

$$
\frac{n^{2}}{\mathbb{R}}+\left\{\frac{\int_{\Sigma} \Gamma \cdot\left(\mathbb{R}^{\varepsilon}-2 \xi_{(\beta+\gamma)}\right)^{\dagger}}{n \Delta^{2}}\right\} \simeq \frac{\mathbb{R}}{n}\left(\Gamma_{(2-\gamma)} \cdot \Delta^{2}\right)+\beta
$$

Now that we have the integration of our output connections reaching fluent and efficient stability for $\mathbb{R}$, we can see that our first output source has a precise reading $\ddagger$ with the help of the accurate output readings from $\beta$ s second output source. Koshlyakov (1964), made this methodology possible by giving a clear and thorough understanding of second order partial differential equations and now we can formulate a better understanding of how the Quantum states work [9].

$$
n \approx \int_{\Sigma} \frac{\mathbb{R}^{2}}{n}+\left(\Gamma^{2}-\Delta\right) \beta \mathrm{d} \Gamma
$$

\section{Stable Output Source of the Input Connections Has Been Achieved}

We can now clearly see that after much stability balancing between both output connections $\Delta$ with much respect to $n, \Gamma$ has found the best stable output connection readings for $\mathbb{R}$ and with $\beta$ s output source connections for:

$$
\begin{gathered}
\Gamma+[\beta]\left(\frac{\Sigma(n)}{\mathbb{R}^{2}}\right) \text { with respect to } \Gamma^{2(\mathbb{R})} \beta \text { as seen in Expression (12). } \\
\qquad \int_{\Sigma} \frac{\mathbb{R}^{2}}{n}+\left(\Gamma^{2}-\Delta\right) \beta \equiv \Gamma+[\beta]\left(\frac{\Sigma(n)}{\mathbb{R}^{2}}\right) \equiv \Gamma^{2(\mathbb{R})} \beta
\end{gathered}
$$

1.4 Theorem: Since $n$ has shown the effectiveness of both $\mathbb{R}$ and $\beta$, we can clearly see the accuracy between our input and output sources that are feeding off of the connections simultaneously and with the fluency of both output readings that lead back to our main tensor connection $\Gamma^{2(\mathbb{R})} \beta$ without any latency as seen above in Expression (13). This is our final answer.

\section{Conclusions}

It was Gregorio Ricci who first invented tensor calculus and Pierre de Fermat 
who invented number theory. But it became very interesting when Quantum Mechanics grabbed a chair to sit next to his friends, "Tensor Calculus and Number Theory". Now we came up with new mathematical expressions and notations. It's the very same reason why I was inspired to do this paper and especially the mathematical Expressions of (1)-(13) in which I used some tensor product applications but mostly number theory to prove the connections of both input and output scenarios. I made sure my methods of explanations are as clear and concise as possible for the reader. I would also like to mention on a side note that I'm hoping this manuscript will be possibly used in applied mathematics and applied physics in the near future. To back up my hopefulness, the reason being is because you can yield results with my formula using applied mathematics. Not just for finding the input and output sources of a Quantum network. Here's a great example of what I'm talking about. My equation formula: $\Gamma^{2(\mathbb{R})} \beta$ used as an applied mathematical formula would look like this:

$7^{2(3)} 4=7^{(6)} 4=(117649) 4=470596$. This is our answer.

It can also be written as: $\left(7^{2(3)}\right) \cdot 4=470596$ to make the process quicker but still yielding the same answer as the first formula that I presented. I firmly believe my formula could be used for several other mathematical applications. I take pleasure in the creation of this paper. Thank you for reading.

\section{Conflicts of Interest}

The authors declare no conflicts of interest regarding the publication of this paper.

\section{References}

[1] Neugebauer, O. (1969) The Exact Sciences in Antiquity. 36-40. http://store.doverpublications.com/0486223329.html

[2] Hua, L.-K. and Wang, Y. (1981) Applications of Number Theory to Numerical Analysis. Springer, Berlin, Heidelberg, 30-220.

[3] Hassani, M. (2003) Derangements and Applications. Journal of Integer Sequences (JIS), 6, 123-126.

[4] Bombieri, E. (1990) The Mordell Conjecture Revisited. Annali della Scuola Normale Superiore di Pisa, Classe di Scienze, 17, 615-640.

[5] Halmos, P.R. (1974) Finite Dimensional Vector Spaces. Springer, Berlin, 24-25. https://doi.org/10.1007/978-1-4612-6387-6

[6] Faltings, G. (1994) The General Case of S. Lang's Conjecture. In: Cristante, V. and Messing, W., Eds., Perspectives in Mathematics, Academic Press, San Diego, CA, 9-10.

[7] Aguiar, M. and Mahajan, S. (2010) Monoidal Functors, Species and Hopf Algebras. In: CRM Monograph Series, Vol. 29, American Mathematical Society, Providence, 33-35. https://doi.org/10.1090/crmm/029

[8] Grillet, P.A. (2007) Abstract Algebra. Springer, Berlin, 43-104.

[9] Koshlyakov, N.S., Smirnov, M.M. and Gliner, E.B. (1964) Differential Equations of Mathematical Physics. 3rd Edition, John Wiley and Sons, New York, 19-80. 